\title{
Hepatitis B in Pregnancy: Challenges and Solutions
}

\author{
Dalia Rafat* and Seema Hakim \\ Department of Obstetrics \& Gynaecology, Aligarh Muslim University, India
}

Submission: November 02, 2016; Published: November 22, 2016

*Corresponding author: Dalia Rafat, Department of Obstetrics \& Gynaecology, J N Medical College \& Hospital, Faculty of Medicine, Aligarh Muslim University, Aligarh, UP, India, Tel: 00919411979687; Email: drdaliarafat.16@gmail.com

Keywords: Hepatitis B virus; Pregnancy; Vertical transmission; Immunoprophylaxis; Antiviral therapy

\section{Introduction}

Hepatitis B is the most common serious liver infection in the world, caused by the hepatitis B virus (HBV), which attacks liver cells and can lead to liver failure, cirrhosis (scarring) or cancer of the liver later in life. According to the World Health Organization (WHO) estimates, two billion people have been infected worldwide with the HBV, which accounts for one-third of the global population, and more than 240 million are chronic carriers (4\%-6\% of the world population). Approximately 600000 people die every year due to the consequences of HBV infection [1].

Modes of transmission include both horizontal spread via contact with infected blood or body fluids and vertically via mother to child transmission (MTCT). A great majority of the transmission of HBV in both high and low endemic areas occurs by vertical transmission from an infected carrier mother to the neonate, during pregnancy or in perinatal periods [2-4].

HBV infection during pregnancy is associated with a high risk of maternal complications, has a high rate of vertical transmission causing fetal and neonatal hepatitis and has been reported as a leading cause of maternal mortality. Also, the risk of progression to chronic HBV infection is inversely proportional to the age at which the infection was acquired and vertical transmission of HBV from infected mothers to their fetuses or newborns remains a major source of perpetuating the reservoir of chronically infected individuals. Therefore, proper management of infected mothers and effective prevention of MTCT is a promising efficient approach to interrupt chronic HBV infection [5].

Management of HBV infection in pregnancy is challenging because of several peculiar and somewhat controversial aspects associated with it, including the following:
A. The effect of pregnancy on HBV infection and of HBV infection on pregnancy.

B. The potential viral transmission from mother to newborn despite at-birth prophylaxis with immunoglobulin and vaccine

C. Possible prevention of mother-to-child transmission through antiviral drugs

D. The type of antiviral drug to use considering their efficacy and potential teratogenic effect, and the timing of their administration and discontinuation

E. The use of elective caesarean section vs vaginal delivery in pregnant women with hepatitis B; and

F. The possibility of breastfeeding.

This document will briefly discuss the solution to these challenges which in turn will aid in better understanding of the clinical, therapeutic, and prognostic aspects of HBV infection during pregnancy.

\section{Effect of HBV Infection on Pregnancy}

Pregnant women with acute or chronic HBV infection typically have a course not very different from that in the general adult population and often no serious effects due to HBV infection are found in pregnancy [6]. Slightly higher incidence of hyper emesis gravid arum, low birth weight and prematurity has been reported in some patients with acute and chronic HBV infection than in the general population [4]. However, mothers with seriously abnormal liver function are prone to postpartum hemorrhage, puerperal infection, fetal distress, fetal death and neonatal asphyxia [7-10]. Patients with advanced cirrhosis are 
at increased risk of maternal and fetal problems in about 50\% of cases [11].

\section{Effect of Pregnancy on HBV Infection}

Series of physiological changes in pregnancy, including vigorous metabolism and increased nutrient consumption occurs to promote the metabolic needs of the mother as well as of the growing fetus. Aggravation of pre-existing liver diseases and exacerbation of liver damage might occur owing to the need of augmented metabolism and inactivation in the liver of the increased sex hormone produced by the mother and dependence of fetal metabolism and detoxification on the mother's liver $[12,13]$. During pregnancy there also occur several modifications in the maternal immune system that contribute to a depressed immune response against $\mathrm{HBV}$ resulting in an increase in HBV DNA and a reduction in aminotransferase levels. After delivery, with restoration of immune system opposite consequences occurs; namely, a significant increase in alanine aminotransferase (ALT) and a reduction of HBV DNA in this period [12]. These modifications may result in viral exacerbations and higher incidence of complications. Although HBV infection during pregnancy can often be tolerated, severe hepatitis and hepatic failure induced by perinatal hepatic flare reactions still occur, and can have an unfavourable outcome [14]. Close monitoring and timely interventions thus are recommended.

\section{Perinatal Transmission}

Perinatal transmission is the most common mode of HBV transmission worldwide, accounting for one third of HBV infections. Because infection with HBV acquired prenatally often leads to chronic disease, prevention of vertical transmission is a worthy goal. Perinatal transmission of HBV is mediated via three main modes:

1. Intrauterine (transplacental transmission of HBV in utero);

2. Intrapartum (transmission during delivery);

3. Postnatal (transmission during care or through breast milk) [5,15-16].

\section{Intrauterine transmission}

Intrauterine transmission of $\mathrm{HBV}$ is proposed to be the most important reason for the failure of passive-active immunoprophylaxis in preventing MTCT [7,9]. Although different diagnostic criteria [17-19] have been considered for the diagnosis of HBV intrauterine infection, till now no consensus have been formed on and the exact mechanism of intrauterine transmission of HBV still remains to be illuminated. The most frequently mentioned hypotheses involve: serum/body fluid transmission, cellular transmission and genetic transmission [20-22]. Known risk factors predisposing to intrauterine infection include maternal $\mathrm{HBeAg}$ positivity, detectable $\mathrm{HBV}$ DNA, specific allelic mutations in maternal HBV, a history of threatened preterm labour, and acute hepatitis B acquired in pregnancy, particularly during the last trimester [23,24].

\section{Intrapartum transimission}

Transmission of HBV during delivery is the most common method of vertical transmission. It is predominantly due to contact of the newborn with the mother's infected secretions or blood at the time of delivery [25].

\section{Postnatal transmission}

Transmission of HBV postnatally may occur in as high as $34 \%$ of cases owing to close contact of the baby with the mother $[25,26]$. However, breastfeeding is not considered as a risk factor for HBV infection [25]. Without immune prophylaxis the risk of mother-to-child transmission is very high. It varies with the HBeAg status of mothers, being highest in HBsAg- and HBeAgpositive mothers (transmission rate of 70\%-90\%) and low for HBsAg-positive $\mathrm{HBeAg}$-negative mothers (transmission rate of 10\%-40\%) [6,25]. A combination of active and passive immune prophylaxis is the optimum strategy to prevent HBV infection in babies of HBsAg positive mothers. Passive immunoprophylaxis consists of the administration of hepatitis $B$ immune globulin (HBIG) whereas active immunoprophylaxis is the administration of hepatitis $B$ vaccine. Although passive-active immunoprophylaxis in infants of HBV-infected mothers has been demonstrably effective in reducing perinatal transmission, a small percentage of infants still become HBV chronic carriers and presumably this is because HBV exposure can occur in utero prior to delivery. These facts adds to the concern that in some cases, correct passive-active immunoprophylaxis given at the time of birth may not prevent infection in those born already infected and further supports the need to explore other strategies like antiviral drugs or HBIG to the mother and mode of delivery. Till now there is no consensus about the therapy choice for pregnant women who are HBsAg-positive and highly viremic in the third trimester to prevent perinatal transmission. Although in such cases, some studies have shown reduction in the risk of vertical transmission by third trimester prophylaxis with antiviral drugs like telbivudine or tenofovir but large, appropriately designed trials to recognize the best drug for this use are still pending [27].

\section{Treatment of HBV During Pregnancy}

Prior to considering treatment of HBV infection during pregnancy, indication of treatment and the risks and benefits must be weighed carefully. Firstly it is important to decide whom to treat. HBV infection in pregnancy can either be acute or chronic. Pregnant women acutely infected with HBV generally don't require antiviral unless there is evidence of acute liver failure. These patients are managed conservatively but should be monitored closely. Those infected chronically, indications for treatment includes chronic chronic liver disease in mother and prevention of vertical transmission in baby [28]. Next major issue is the consequences of the treatment both for the mother and the 
fetus. Seven drugs have been approved by the United States Food and Drug Administration (FDA) for the treatment of hepatitis B: PEG-interferon alpha $2 \mathrm{a}$, interferon alpha $2 \mathrm{~b}$, lamivudine, adefovir, entecavir, telbivudine and tenofovir [29]. Interferon's are contraindicated in pregnancy. Among rest five antiviral drugs all of them are category $\mathrm{C}$ except for last two namely telbivudine and tenofovir, which are category B for pregnancy. Safety data in pregnancy are most robust with lamivudine and tenofovir compared with other therapies [29,30]. Finally, if the decision has been made to treat with antiviral, the next worthy consideration is the goal of treatment and the timing of administration of these medications. For pregnant women who develop active liver disease the goal of treatment should be to induce liver disease remission in the mother, in order to minimize the risk of preterm delivery while for others the goal should be to prevent transmission of HBV at birth by decreasing viral load and/or decreasing activity of the virus [29-31]. Although still controversial, but most studies have shown that if indicated antivirals should be started in the third trimester of pregnancy [31-33]. Careful discussion with the patient regarding the risks and benefits of therapy is warranted.

\section{Mode of Delivery}

There is conflicting data in literature over the effect of mode of delivery on perinatal HBV transmission [34,35]. Although the mode of delivery to maximally reduce the incidence of vertical transmission of HBV still remains controversial but measures available to reduce the infection rate of HBV in labor should be opted in all cases. Currently, there is no convincing evidence that elective caesarean section reduces the rate of mother-to-child transmission of HBV compared with vaginal delivery and so most obstetric societies do not endorse routine use of caesarean section to prevent perinatal transmission of HBV.

\section{Breastfeeding by HBV-Infected Women}

Transmission of HBV via breast milk is frequently an added concern of HBV infected mothers. Currently, the presence of HBsAg, HBeAg and HBV DNA in breast milk is confirmed but the benefits of breastfeeding outweigh the potential risk of infection, which is minimal. In addition, with universal immunoprophylaxis at birth, any potential risk is further reduced. According to current guidelines, all women with hepatitis B should be encouraged to breastfeed their newborns [36]. For mothers on antiviral therapy, breastfeeding is not recommended because few data are available about the safety of antiviral exposure during breastfeeding [37].

\section{Conclusion}

HBV infection in pregnancy is a challenging condition, but providing solutions to these challenges is noteworthy and an important opportunity to interrupt perinatal transmission of HBV. Since maternal-fetal transmission is the major route of acquisition of HBV worldwide and age of acquisition of HBV infection is directly proportional to chronicity of disease, strategies to eradicate HBV or to decrease global burden of disease must aim this critical step in HBV disease propagation. Testing for HBsAg is recommended for every pregnant woman, regardless of previous testing or vaccination. Standard passiveactive immunoprophylaxis with HBIG plus HBV vaccine in neonates within $12 \mathrm{~h}$ after delivery is proved to be successful in preventing approximately $90 \%$ of vertical transmission of HBV. However, in the cases of high maternal viremia, a small proportion of newborns can acquire the infection (probably through in utero transmission) despite the use of passive/active prophylaxis. In such situations, antiviral treatment in the third trimester can be considered. Administration of antiviral therapy is also needed in those who have active liver disease during pregnancy.

The choice of antiviral should be restricted to those drugs considered safe in this setting and the risks and benefits of treatment must be weighed carefully. Chronic HBV infection does not seem to increase risk of maternal fetal morbidity and mortality. Additionally, standard vaginal delivery and breastfeeding do not appear to increase the risk of HBV transmission.

There are still some controversial issues that require further concerns, including HBIG injection during pregnancy, breastfeeding of infants by mothers on antiviral therapy and the potential long-term side effect of antiviral agents to both HBV positive mothers and their infants. These challenges still need solutions and calls for further research in this field.

\section{References}

1. World Health Organization (2013) Hepatitis B. Media Centre, Switzerland.

2. Goyal A, Murray JM (2014) the impact of vaccination and antiviral therapy on hepatitis B and hepatitis D epidemiology. PLoS One 9(10): e110143.

3. Lavanchy D (2005) worldwide epidemiology of HBV infection, disease burden, and vaccine prevention. J Clin Virol 34 (Suppl 1): S1-S3.

4. Jonas MM (2009) Hepatitis B and pregnancy: an underestimated issue, Liver Int 29(Suppl 1): 133-139.

5. Ma L, Alla NR, Li X, Mynbaev OA, Shi Z (2014) Mother-to-child transmission of HBV: review of current clinical management and prevention strategie. Rev Med Virol 24(6): 396-406.

6. Borgia G, Carleo MA, Gaeta GB, Gentile I (2012) Hepatitis B in pregnancy. World J Gastroenterol 18(34): 4677-4683.

7. Sinha S, Kumar M (2010) Pregnancy and chronic hepatitis B virus infection. Hepatol Res 40(1): 31-48.

8. Kwon CI, Hwang SG, Shin SJ, Chang SW, Kim SY, et al. (2008) Occult hepatitis $B$ virus infection in pregnant woman and its clinical implication. Liver Int 28(5): 667-674.

9. Wong HY, Tan JY, Lim CC (2004) Abnormal liver function tests in the symptomatic pregnant patient: the local experience in Singapore. Ann Acad Med Singapore 33(2): 204-208.

10. Tse KY, Ho LF, Lao T (2005) the impact of maternal HBsAg carrier status on pregnancy outcomes: a case-control study. J Hepatol 43(5): 771-775.

11. Hay JE (2008) Liver disease in pregnancy. Hepatology 47(3):10671076. 
12. Borg MJ, Leemans WF, de Man RA, Janssen HL (2008) Exacerbation of chronic hepatitis B infection after delivery. J Viral Hepat 15(1): 37-41.

13. Buster EH, van Erpecum KJ, Schalm SW, Zaaijer HL, Brouwer JT, et al. (2008) Treatment of chronic hepatitis B virus infection - Dutch national guidelines. Neth J Med 66(7): 292-306.

14. Lin HH, Chen PJ, Chen DS, Sung JL, Yang KH, et al. (1989) Postpartum subsidence of hepatitis B viral replication in $\mathrm{HBeAg}$-positive carrier mothers. J Med Virol 29(1): 1-6.

15. Hou J, Liu Z, Gu F (2005) Epidemiology and prevention of hepatitis B virus infection. Int J Med Sci 2(1): 50-57.

16. Navabakhsh B, Mehrabi N, Estakhri A, Mohamadnejad M, Poustchi H (2011) Hepatitis B virus infection during pregnancy: transmission and prevention. Middle East J Dig Dis 3(2): 92-102.

17. Goudeau A, Yvonnet B, Lesage G, Barin F, Denis F et al. (1983) Lack of anti-HBc IgM in neonates with HBsAg carrier mothers argues against transplacental transmission of hepatitis $B$ virus infection. Lancet 2(8359): 1103-1104.

18. Xu DZ, Yan YP, Choi BC, Xu JQ, Men K et al. (2002) Risk factors and mechanism of transplacental transmission of hepatitis B virus: a case-control study. J Med Virol 67(1): 20-26.

19. Xu YY, Liu HH, Zhong YW, Liu C, Wang Y, et al. (2015) Peripheral blood mononuclear cell traffic plays a crucial role in mother-to-infant transmission of hepatitis B virus, Int J Biol Sci 11(3): 266-273.

20. Bai H, Zhang L, Ma L, Dou XG, Feng GH (2007) Relationship of hepatitis $B$ virus infection of placental barrier and hepatitis B virus intra-uterine transmission mechanism. World J Gastroenterol 13(26) 3625-3630.

21. Towers CV, Asrat T, Rumney P (2001) the presence of hepatitis B surface antigen and deoxyribonucleic acid in amniotic fluid and cord blood. Am J Obstet Gynecol 184(7): 1514-1518.

22. Yu M, Jiang Q, Gu X, Ju L, Ji Y, et al. (2013) Correlation between vertical transmission of hepatitis B virus and the expression of HBsAg in ovarian follicles and placenta. PLoS One 8(1): e54246.

23. Lv N, Chu XD, Sun YH, Zhao SY, Li PL, et al. (2014) Analysis on the outcomes of hepatitis $\mathrm{B}$ virus perinatal vertical transmission: nested case-control study. Eur J Gastroenterol Hepatol 26(11): 1286-1291.

24. Pan CQ, Duan ZP, Bhamidimarri KR, Zou HB, Liang XF et al. (2012) An algorithm for risk assessment and intervention of mother to child transmission of hepatitis B virus. Clin Gastroenterol Hepatol 10(5): 452-459.

25. Piratvisuth T (2013) optimal management of HBV infection during pregnancy. Liver Int 33 (Suppl 1):188-194.

26. Degli Esposti S, Shah D (2011) Hepatitis B in pregnancy: challenges and treatment. Gastroenterol Clin North Am 40(2):355-372.

27. Gentile I, Borgia G (2014) Vertical transmission of hepatitis B virus: challenges and solutions. Int J Womens Health 6: 605-611.

28. Degertekin B, Lok AS (2009) Indications for therapy in hepatitis B. Hepatology 49(5 suppl): S129-S137.

29. Tran TT (2009) Management of hepatitis B in pregnancy: weighing the options. Cleve Clin J Med 76(Suppl 3): S25-S29.

30. Marcellin P, Heathcote EJ, Buti M, Gane E, de Man RA, et al. (2008) Tenofovir disoproxil fumarate versus adefovir dipivoxil for chronic hepatitis B. N Engl J Med 359 (23): 2442-2455.

31. Pan CQ Mi LJ, Bunchorntavakul C, Karsdon J, Huang WM (2012) Tenofovir disoproxil fumarate for prevention of vertical transmission of hepatitis B virus infection by highly viremic pregnant women: a case series. Dig Dis Sci 57(9): 2423-2429.

32. Han GR, Cao MK, Zhao W, Jiang HX, Wang CM (2011) A prospective and open-label study for the efficacy and safety of telbivudine in pregnancy for the prevention of perinatal transmission of hepatitis $B$ virus infection. J Hepatol 55(6): 1215-1221.

33. Zonneveld VM, van Nunen AB, Niesters HG, de Man RA, Schalm SW (2003) Lamivudine treatment during pregnancy to prevent perinatal transmission of hepatitis B virus infection. J Viral Hepat 10(4): 294297.

34. Wang J, Zhu Q, Zhang X (2002) Effect of delivery mode on maternalinfant transmission of hepatitis B virus by immunoprophylaxis. Chin Med J (Engl) 115(10): 1510-1512.

35. Yang J, Zeng XM, Men YL, Zhao LS (2008) Elective caesarean section versus vaginal delivery for preventing mother to child transmission of hepatitis B virus--a systematic review. Virol J 5: 100.

36. Lok AS, McMahon BJ (2009) chronic hepatitis B: update 2009. Hepatology 50(3): 661-662.

37. Bzowej NH (2010) Hepatitis B therapy in pregnancy. Curr Hepat Rep 9(4): 197-204.

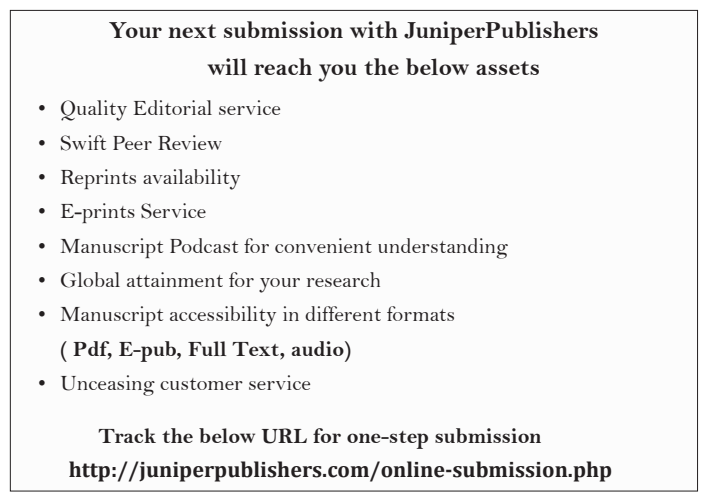

